CHAPTER 8

\title{
Treatment of lupus nephritis
}

\author{
Sebastian Dolff ${ }^{1,2}$, Jo H.M. Berden ${ }^{3}$, Marc Bijl
}

\footnotetext{
${ }^{1}$ Department of Rheumatology and Clinical Immunology, University Medical Center Groningen, University of Groningen, The Netherlands

${ }^{2}$ Department of Nephrology, University Hospital Essen, University Duisburg-Essen, Germany

${ }^{3}$ Department of Nephrology, University Medical Centre St Radboud Nijmegen,

Radboud University Nijmegen, The Netherlands
}

Expert Rev Clin Immunol. 2010 Nov; 6 (6): 901-11. 
Abstract

Renal involvement in systemic lupus erythematosus patients (SLE) is a severe disease manifestation characterized by various clinical and histopathological alterations. The revised ISN/RPN 2003 classification defines the subclasses of lupus nephritis (LN) according to their pathological glomerular patterns, which has a crucial impact on the prognosis and treatment options in $L N$ patients. There are widely accepted therapeutic agents such as cyclophophamide, mycophenolate mofetil, azathioprine and corticosteroids available. Several trials tried to determine a gold standard for the induction and maintenance therapy in LN and the place of newer drugs, biologicals, is investigated. We review recently reported data on current treatment regimens in $L N$ in particular in the context of the ISN/RPN 2003 classification. 


\section{Introduction}

Systemic lupus erythematosus (SLE) is an autoimmune disease that is characterized by the production of autoantibodies to nuclear components. ${ }^{1}$ The inflammatory response triggered by in situ formation and/or deposition of immune complexes is thought to be responsible for the various clinical manifestations such as vasculitis, nephritis and skin involvement. Especially, renal involvement, i.c. lupus nephritis (LN), remains a frequent and severe manifestation of SLE. Renal involvement in SLE is still one of the strongest predictors for morbidity and mortality. ${ }^{2-4}$ Although over the last decades the therapeutic options for LN have increased leading to better results, the survival rate among patients with $L N$ is still about $75 \%$ after 15 years. ${ }^{2}$

The underlying mechanisms in the pathogenesis of $L N$ are still poorly understood. Numerous immunological abnormalities have been found to be associated with LN. However, which factors relate directly to renal inflammation remains unclear. Renal involvement in human SLE has often been considered as a classical immune complex induced glomerulonephritis (GN) due to deposition of preformed circulating immune complexes or binding of autoantibodies to glomerular antigens. Characteristic autoantibodies such as anti-dsDNA, but also antibodies with specifity for nucleosomes, Sm, histone, SS-A or SS-B have been found in kidney eluates from murine lupus models and from LN patients..$^{5-7}$ Moreover, it has been demonstrated that anti-DNA antibodies can also interact with glomerular constituents such as endothelial cells, type IV collagen, a-actinin-4 and heparan sulfate supporting the hypothesis of an immune complex mediated disease. ${ }^{8 ; 9}$ Together, this data provides further evidence that autoantibodies play a crucial role in the pathogenesis of $L N$.

Therefore, B cells, as the source of antibodies, have been acknowledged as an important lymphocyte subset in the pathogenesis of SLE and $L N$ in particular. Alterations of $B$ cell numbers, activation status and function have been demonstrated. At first glance, B cells, being the source of autoantibodies, are regarded as the main actors in the immunological cascade 
ending in organ injury. There is growing evidence that, besides autoantibody secretion, other functions of $B$ cells such as antigen presenting capacity and cytokine production contribute to the development of SLE as well. Since the autoimmune response is autoantigen driven and T-cell dependent, also T cells are involved. For the interaction between B- and T-cells ligation of costimulatory molecules is necessary. ${ }^{10}$ The increased expression of costimulatory molecules on B- and T-cells in SLE suggested novel therapeutic targets. ${ }^{11 ; 12}$ However, Tcells do more than initiation of B-cell activation. They can exert effector cell function themselves. The presence of T-cells in renal biopsies of LN patients and their presence in urine during active renal disease, support their migratory abilities and potential pathogenic role. ${ }^{13 ; 14}$

Because of the described involved immunological mechanisms, the therapeutic regimens in SLE consist mainly of immunosuppressive drugs. Several therapeutic options are nowadays available to induce and maintain remission. However, therapy related toxicity is still a major concern in this, overall relatively young, patient group. This manuscript will review the current status of the therapeutic options in LN.

\section{Clinical features of lupus nephritis}

Renal manifestations in SLE are frequent and may vary from mild to severe. Almost $50-60 \%$ of all SLE-patients develop glomerulonephritis during the course of their disease. Therefore, early detection and monitoring of renal involvement is crucial. Evaluation for LN includes dipstick and microscopic urinanalysis, urinary protein and creatinine excretion, determination of serum creatinine and serological markers such as anti-dsDNA antibody titres and C3 and C4 levels. All of these parameters are taken into account when treatment is designed. Several studies examined the correlations of serological abnormalities with renal histology. Serum C3 and anti-dsDNA titers have been considered as valuable predictors. ${ }^{15-17}$ However, none of the clinical or serological parameters, either separately or in combination, can adequately predict the specific histological abnormalities and severity of the renal injury in 140 
SLE patients. Therefore, a renal biopsy is mandatory. Thus, all patients suspected to have LN should undergo a renal biopsy, unless there are contraindications.

Treatment of LN requires an understanding of the immunopathology. Therefore, we will briefly discuss the histopathological findings, which are characteristic for $L N$ and are the basis for the ISN/RPS classification (Table 1). ${ }^{18}$

ISN/RPS Classification of lupus nephritis (LN) (2003)

\begin{tabular}{ll}
\hline class I & minimal mesangial $L N$ \\
class II & mesangial proliferative $L N$ \\
class III & focal $L N(<50 \%$ of all glomeruli) \\
class IV & diffuse $L N(>50 \%$ of all glomeruli) \\
class V & membranous $L N$ \\
class VI & advanced sclerosing $L N$
\end{tabular}

Table 1. ISN/RPS classification of lupus nephritis (2003).

\section{Histopathology}

The histopathological findings are currently categorized by the ISN/RPS classification. The International Society of Nephrology (ISN) and the Renal Pathology Society (RPS) elaborated this classification in 2003 as a modification of the original WHO classification of 1974 (Table 1). ${ }^{18}$ The classification comprises six classes. The classification is based entirely on evaluation of glomerular alterations, although tubular, interstitial and vascular lesions are frequent findings in LN. Glomerular alterations are characterized by glomerular hypercellularity in the mesangial, endocapillary or extracapillary areas. Class I represents the mildest form of LN. Light microscopy reveals normal-appearing glomeruli, immunofluorescence microscopy however, shows mesangial immune deposits. Class II LN is defined as pure mesangial proliferation by light 
microscopy. Additionally, subendothelial or subepithelial deposits can be visible by immunofluorescence or electron microscopy. If there are on light microscopy endo- and/or extracapillary alterations visible, LN has to be classified as Class III or IV depending on the severity and distribution. In Class III, the focal lesions are typically segmental and involve less than $50 \%$ of glomeruli. Class IV is defined as diffuse segmental and/or global endocapillary and/or extracapillary glomerulonephritis affecting more than or equal to $50 \%$ of the glomeruli. Furthermore is class IV subdivided into two categories to define whether the proliferative lesions are segmental (IV-S) or global (IV-G). If less than $50 \%$ of the glomerular tuft is affected it is designated as IV-S and as IV-G if $\geq 50 \%$ of the glomerular tuft is involved. Both class III and IV may have purely active (A), chronic $(C)$ or mixed active and chronic lesions $(A / C)$, which is indicated by the addition of the capital letters $A$ and $C$. Class $V$ defines membranous $L N$ and simplifies the modified WHO classification of 1982 by eliminating the four confusing subclasses Va-Vd. Membranous LN can also be associated with Class III or Class IV. If so, both classes are mentioned ( $\mathrm{V}+$ III or $\mathrm{V}+\mathrm{IV})$. If more than $90 \%$ of glomeruli are sclerotic and without residual inflammatory activity, it is defined as Class VI.

In order to evaluate LN more precisely, most investigators supplement the aforementioned ISN/RPS classification with a semiquantitative grading, developed at the National Institute of Health $(\mathrm{NIH})$, to assess activity (potentially reversible lesions) and chronicity (irreversible lesions). ${ }^{19}$ These histological features, listed in Table 2, have to be graded on a scale of 0-3. Fibroid necrosis and cellular crescents are assigned double weight. The sum of all features evaluating the activity index yields a score of 0 to 24 and for the chronicity index a score of 0-12. Apart from the predictive value of these supplementary indices, the scoring of activity and chronicity provides important information about disease progression over time in case of sequential biopsies. ${ }^{20}$ 
Activity and Chronicity Index Activity

Index of Activity (0-24)

Endocapillary hypercellularity

Leukocyte infiltration

Subendothelial hyaline deposits

Fibrinoid necrosis/karyorrhexis*

Cellular crescents*

Interstitial inflammation

Index of Chronicity (0-12)

Glomerular sclerosis

Fibrous crescents

Tubular atrophy

Interstitial fibrosis

Table 2. The NIH scoring system for the activity and chronicity index is shown. Each item is graded on a scale of $0-3$. The more severe lesions $\left(^{*}\right)$ are multiplied by 2 . The sum of all features yields a score for the activity index between 0-24 and for the chronicity index between 0-12.

The understanding of the classification of $L N$ has a major impact on the analysis of past and present studies evaluating treatment strategies. Due to the heterogeneous nature of $L N$, most studies enroll a selective cohort of patients based on the histopathological findings. Therefore, the therapeutic options will be discussed for the different classes of $L N$.

\section{Immunosuppressive treatment of lupus nephritis \\ Class I lupus nephritis}

In general there is no therapy necessary in patients with Class I LN in order to treat renal involvement (Figure 1). Administration of immunosuppressive regimens is dependent on extra-renal manifestations and disease activity. Patients should be monitored carefully and regularly to detect at an early stage transition to a more severe class of LN. 


\section{Class II lupus nephritis}

In patients with mesangial proliferative Class II LN corticosteroids might be administrated to prevent disease progression and development of a class III or IV LN. Along with supportive treatment such as ACE-inhibitors in patients with proteinuria $(>1.0 \mathrm{~g} / 24 \mathrm{~h})$ and/or active urinary sediment, prednisone can be given $(0.5-1 \mathrm{mg} / \mathrm{kg} / \mathrm{day}$, tapered to 0 over six months). Due to the lack of controlled studies, this recommendation is purely based on expert opinion. In addition, treatment with hydroxychloroquine can be considered. ${ }^{21}$ Also in patients with Class II LN, regular monitoring of disease activity and renal parameters is mandatory.

\section{Class III/IV lupus nephritis}

Class III and IV LN have several pathogenic and histopathological similarities and patients with these types of $L N$ share a poor long term renal survival due to deterioration of kidney function. Therefore, clinical trials usually comprise both groups. The first therapeutic goal is to reduce disease activity and to restore renal function or to prevent deterioration. Therefore, an aggressive treatment strategy (corticosteroids and cytotoxic drugs), so called induction therapy, is necessary for a period of time between 6 and 24 months. After successful induction therapy, long-term maintenance therapy is advocated to prevent renal relapses. ${ }^{22}$

\section{Induction therapy}

As induction therapy high dose intravenous cyclophosphamide (ivCy) in combination with high dose prednisone dominated for years treatment of LN, since long-term outcome, especially in terms of preservation of renal function, was superior over treatment with prednisone alone. ${ }^{23 ; 24}$ In these randomized controlled trials (RCTs) SLE patients received monthly for up to 6 months, and then every 3 month for another 18 months infusions of cyclophosphamide $500 \mathrm{mg}$ to $1000 \mathrm{mg}$ per square meter $\left(\mathrm{m}^{2}\right)$ of body surface. The occurrence of end-stage renal disease (ESRD) was significantly lower compared to patients 
receiving oral prednisone alone $(1.0 \mathrm{mg} / \mathrm{kg}$ body weight for $4-8$ weeks, then tapered). Notably, this difference could only be detected after a follow up of at least 5 to 6 years. The survival rate did not differ between these groups in these studies. Nevertheless, these data demonstrated a beneficial effect of high dose ivCy on top of prednisone and this combination has been the gold standard for the therapy of proliferative $L N$ for a long time. Patients with severe renal impairment (GFR<80 ml/min.) and/or $>25 \%$ of glomeruli affected by cellular crescents had a lower probability of doubling serum creatinine when treated with long-course ivCy $\left(0.5-1.0 \mathrm{~g} / \mathrm{m}^{2}\right.$, every month for 6 months followed by quarterly pulses cyclophosphamide for 2 additional years) in comparison to intravenous methylprednisolone alone $\left(1.0 \mathrm{~g} / \mathrm{m}^{2}\right) .{ }^{25}$ However, the use of ivCy resulted in high toxicity. Upper airway infections, herpes zoster infections and amenorrhea have been frequently reported. ${ }^{24}$

The Euro-Lupus Nephritis Trial (ELNT) was initiated to test whether good long-term clinical results could also be achieved using lower doses of ivCy (6 pulses of $500 \mathrm{mg}$ given at intervals of 2 weeks) versus a higher dose regimen (6 monthly pulses and 2 quarterly pulses with escalating doses, starting with $500 \mathrm{mg}$, increased by $250 \mathrm{mg}$ each course to maximum of $1500 \mathrm{mg}$ per pulse), each of which was followed by azathioprine. ${ }^{26}$ Patients with biopsy proven proliferative glomerulonephritis (WHO class III, IV, Vc or Vd) were recruited between 1996 and 2000. After ten years follow-up there were no significant differences between the low dose and high dose regimen with regard to doubling of serum creatinin, ESRD and patient survival. Of all patients $(n=90)$ $7 \%$ had reached ESRD. ${ }^{27}$ Only $12 \%$ reached sustained doubling of serum creatinine after this period of time. About $70 \%$ of the patients randomised to the low dose arm did not receive any additional cyclophosphamide pulse during a 10 year period. The low dose ivCy regimen had less toxicity. Thus, the low dose ivCy followed by AZA as maintenance treatment seems to be efficient and safe in Caucasian patients according to the ELNT. 
induction maintenance relapse

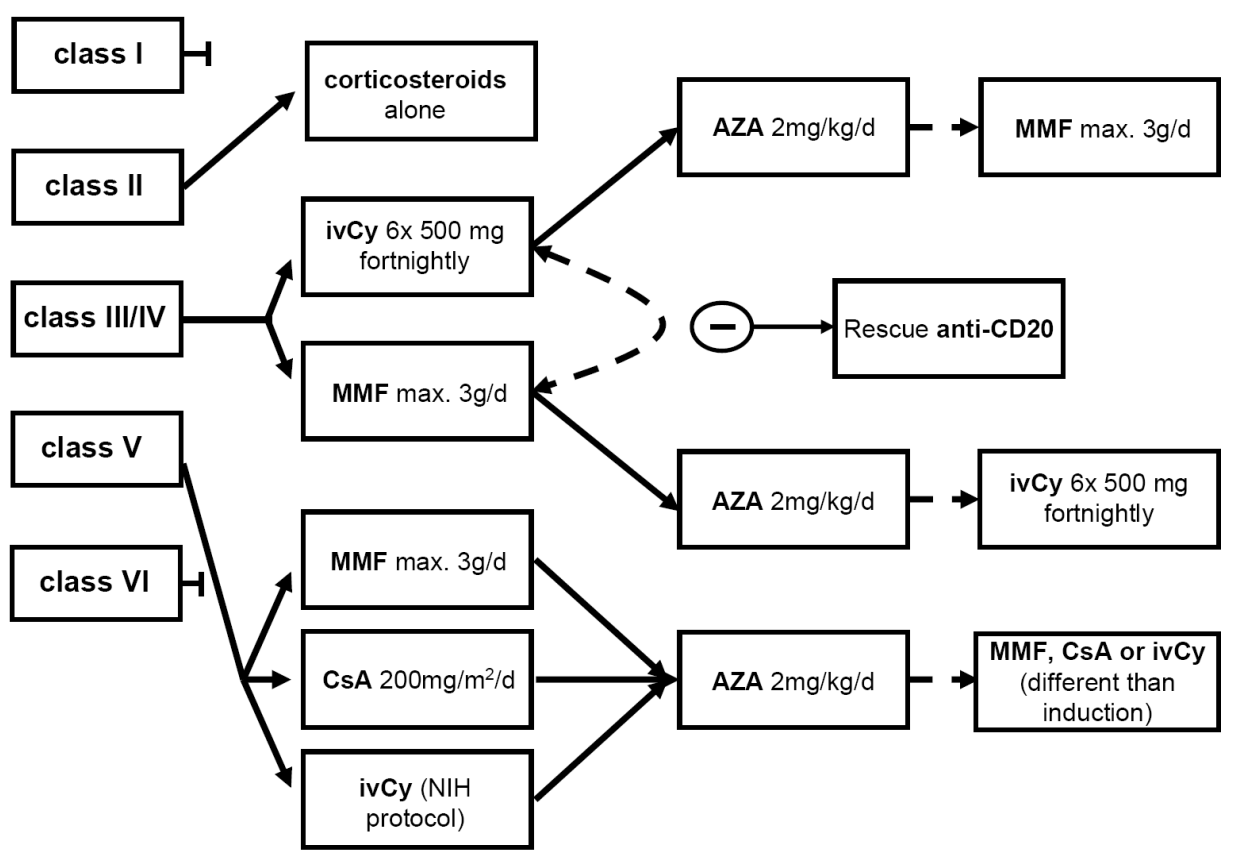

Figure 1. Schematic view of currently recommended therapeutic regimens for the different classes of LN. All immunosuppressive regimens are accompanied with prednisone. The arrows represent responder $(\rightarrow)$ or non responder $(-->)$.

With respect to the acknowledged adverse effects of ivCy, especially associatedwith high dose regimens, other drugs have been studied as alternative induction regimens. The Dutch Working Party on SLE investigated the use of azathioprine $(2 \mathrm{mg} / \mathrm{kg} /$ day $)$ combined with i.v. methylprednisone (MP) and low dose oral prednisone (initially $20 \mathrm{mg}$ ) in comparison to cyclophosphamide pulse therapy $\left(750 \mathrm{mg} / \mathrm{m}^{2}\right)$ in a RCT. In total $87 \mathrm{SLE}$ patients with proliferative LN were included. ${ }^{28}$ The authors reported initially a similar efficacy regarding the proportion of patients that reached the primary end point (non-sustained doubling of initial serum creatinine). However, after a longer follow-up of 6.4 years doubling of serum creatinine (RR 5.2; $\mathrm{Cl}$ : 1.1-25.2; $\mathrm{p}=0.04)$ and renal relapses (RR 4.9; $\mathrm{Cl}: 1-6-15.0 ; \mathrm{p}=0.06)$ occurred more 
frequently in the AZA/MP arm. Furthermore, infectious events, especially herpes zoster infections, were more often seen in the AZA/MP arm. ${ }^{28}$ The clinical observation that the ivCy regimen is superior to AZA was supported by the histopathological assessment of renal biopsies obtained at study entry and after 2 years. $^{29}$ The renal specimens of the patient receiving AZA/MP showed a more pronounced progression of chronic lesions than in the ivCy arm $(p=0.05)$. Taken together, these studies show that the use of AZA/MP as induction therapy might be an option in selected patients, in particular women who do not accept the risk of infertility associated with ivCy.

In recent years mycophenolate mofetil (MMF) was evaluated as induction therapy. Several studies analyzed MMF in comparison to cyclophosphamide as induction therapy in SLE patients with proliferative LN. One of the first was initiated by Chan et al. in $2000 .^{30}$ The use of MMF (2 g/day) versus oral cyclophosphamide $(2.5 \mathrm{mg} / \mathrm{kg} /$ day) followed by AZA was tested in 42 SLE patients with diffuse proliferative LN (WHO class IV). Complete remission was reached in about $80 \%$ of patients in both groups. The proportion of patients which had a relapse or even treatment failure was similar in both groups. The authors concluded from their study that MMF in combination with prednisone is as effective as a regimen of oral Cy in combination with prednisone followed by AZA and prednisone. These comparable results persisted after a longer follow-up of 63 months. ${ }^{31}$ However, MMF was associated with fewer infections $(p=0.013)$. Based on these results larger, open-labelled RCTs were performed. In a 24 week multicenter noninferiority trial, Ginzler el al. compared MMF (1000 mg/day, increased to $3000 \mathrm{mg} /$ day $)$ with 4-weekly ivCy $\left(500 \mathrm{mg} / \mathrm{m}^{2}\right.$ of body surface area, increased to $\left.1000 \mathrm{mg} / \mathrm{m}^{2}\right) .^{32}$ In contrast to the study of Chan et al. a large cohort of 140 patients was recruited including patients with class III, class IV and class V LN. The primary endpoint was complete renal remission (CR) at week 24, defined as the return to within $10 \%$ of normal values for serum creatinine, proteinuria and urinary sediment abnormalities. Due to the strict definition of $\mathrm{CR}$, the proportion of patients which reached CR was low in both groups. However, it 
was significantly higher in the MMF group (23\%) compared with the ivCy group (6 \%, $p=0.05)$. The studies by Chan et al. and Ginzler et al. raised the question whether MMF might even be superior to cyclophosphamide for the treatment of LN. To test this hypothesis a large study on behalf of the Aspreva Lupus Management Study Group, the ALMS study, has been performed. ${ }^{33}$ This study enrolled 370 patients with biopsy proven LN class III, class IV and V. The dosages of MMF and ivCy were similar to the aforementioned study of Ginzler et al. The primary endpoint in this study was a prespecified decrease in urine protein/creatinine ratio and stabilization ( $\pm 25 \%$ of baseline) or improvement in serum creatinine. Overall, the study failed to show superiority of MMF versus the ivCy regimen. About $56 \%$ of patients responded to the MMF treatment in comparison to $53 \%$ in the ivCy arm. Also the secondary endpoints (CR, systemic disease activity and safety) were similar in both groups. Remarkably, a subanalysis of the patients according to their racial background, revealed that those patients who were grouped as "others" (mostly blacks and mixed-race) showed a significantly better therapeutic response in the MMF arm $(60.4 \%)$ in comparison to ivCy $(38.5 \%, p=0.033)$. The other groups, Caucasian and Asian, respectively showed response rates between $53.2 \%$ and $63.9 \%$ but without statistically significant differences between MMF and ivCy. The difference between the Ginzler (percentage blacks $61 \%$ ) and the ALMS study (percentage blacks $26 \%$ ) therefore seems to be the ethnic composition of the studies. All in all, the Ginzler study indicated that MMF was more effective than ivCy with a more favourable safety profile and the ALMS study that MMF was equal to ivCy in induction of remission in LN. In 2008 Bao et al. reported a RCT in patients with LN class IV + V comparing tacrolimus (4 mg/day), and MMF (1000 mg/day) with monthly ivCy $\left(750 \mathrm{mg} / \mathrm{m}^{2}\right)$. Steroids were given in both arms (start 3 times $500 \mathrm{mg}$ methylprednisolon; thereafter $0.6-0.8 \mathrm{mg} / \mathrm{kg}$ with subsequent tapering). ${ }^{34}$ At 6 and 9 months $\mathrm{CR}$ in the tacro/MMF arm was 50 and $65 \%$ respectively, while for the ivCy arm this was 5 and $15 \%$. Extrapolation of these results is hampered by the short follow-up of this study and the fact that only Chinese patients were included. 
In conclusion, the currently available data show that MMF and ivCy have at least equal efficacy for the induction treatment of proliferative LN, but ivCy is associated with more severe adverse effects, particularly infections and infertility. Although MMF has obviously a more favourable profile, a final judgement has to be postponed until long-term data regarding MMF treatment become available. MMF seems to be the preferable therapy especially in blacks and young female patients with pregnancy wishes. ${ }^{35}$ However, MMF can not be used during pregnancy because of its teratogenic effects. Apart from these criteria, the therapy decision can be driven by several other factors such as patients' compliance, drug intolerance or earlier treatment regimens. The choice for induction therapy in an individual patient should take into account all these aspects.

\section{Maintenance therapy}

The prevention of flares and renal relapses is the primary goal of maintenance therapy. Besides a frequent monitoring of the patient including assessment of renal parameters, the choice of long-term therapy is crucial. To achieve this goal ivCy seems to be an inadequate option because of the acknowledged side effects especially with increasing cumulative doses. Although, there is no clear consensus on the nomenclature "long-term" and subsequently no unequivocal recommendation can be given, cyclosporine $A(C s A), A Z A$ and $M M F$ are the most preferable alternatives in combination with corticosteroids. A recent RCT comparing CsA (initial dose $4 \mathrm{mg} / \mathrm{kg}$ per day, reduced to a maintenance dose of 2.5 to $3.0 \mathrm{mg} / \mathrm{kg}$ per day) and AZA demonstrated equal efficacy in the prevention of renal flares in a 24 months follow up for both regimens. ${ }^{36}$ However, one should realize that CsA has less favourable side-effects including nephrotoxicity, hypertension and hyperlipidemia. Contreras et al. demonstrated in a cohort of $59 \mathrm{LN}$ patients that MMF and AZA are more efficacious and safer than long-term therapy with ivCy. ${ }^{37}$ In this study major adverse events such as pneumonia, sepsis and meningitis have been reported in $25 \%$ of the patients receiving ivCy maintenance therapy. This was significantly higher than in the 
AZA group $(p=0.01)$ and the MMF group $(p=0.02)$, respectively. To assess which sequential regimen is superior two pivotal studies are under evaluation or still recruiting (MAINTAIN study, NCT00204022) and the maintenance results of ALMS. The results of these studies will elucidate how long and which dosages should be used in the maintenance regimen of proliferative lupus nephritis. Preliminary results from the MAINTAIN study (mainly Caucasian patients) report after a median follow-up for MMF of 48.4 months and for AZA of 54.3 months an equal efficacy of $A Z A$ and MMF with regard to time to flare, percentage of flares and doubling of serum creatinine.$^{38} \mathrm{~A}$ recent meta-analysis reported no differences between MMF and AZA regarding the occurrence of relapses, renal failure and death. ${ }^{39}$ The incidence of proteinuric flares, changes in proteinuria and GFR were similar in the two treatment groups. In conclusion, at present AZA seems to be the best option for maintenance treatment of $L N$ in Caucasian patients. It remains to be determined whether MMF is the best option for AfroAmericans. Further results of the currently conducted studies are necessary to draw a final conclusion.

There are hardly any data available on safety of discontinuation of immunosuppressive treatment in patients with proliferative LN. However, it seems arguable to taper immunosuppression slowly in patients who have been treated for at least 5 years and who have been serologically and clinically quiescent over a long period of time. ${ }^{22}$

\section{Class V lupus nephritis}

The prevalence of pure membranous glomerulopathy in SLE varies from approximately 10 to $40 \%$ of cases of LN. ${ }^{40}$ Membranous LN has been a difficult entity to evaluate due to lower prevalences reported in the past. The lack of uniformity in the nomenclature also hampers analysis of the literature, in particular prior to 1982. Clinically, the majority of patients with membranous lupus nephropathy present with nephrotic-range proteinuria associated with edema. ${ }^{41 ; 42}$ The characteristically young female population has an increased long-term risk for ESRD. Mercadal et al. observed after 20 years renal survival 150 
in only $50 \%$ of their patients with membranous LN. ${ }^{43}$ The optimal treatment for this entity has been controversial. A recent randomized, controlled trial of prednisone (Pred), intravenous cyclophosphamide (ivCy) or cyclosporine (CsA) by Austin et al. enrolled 42 patients with a median urinary protein excretion rate of $5.4 \mathrm{~g} / \mathrm{d} .{ }^{44}$ The authors reported proteinuria $>5 \mathrm{~g} / \mathrm{d}$ as best clinical baseline predictor associated with a decreased probability of reaching remission. The actual goal of this study was to analyze achievement of remission in the various treatment groups. At 1 year the cumulative probability of remission of proteinuria was $27 \%$ in the prednisone group; $60 \%$ with ivCY and $83 \%$ with CsA. However, the difference between the ivCy and CsA treated patients was statistically not significant. All patients who achieved remission of proteinuria were subsequently treated with low-dose prednisone. Interestingly, although inducing remission with CsA was reasonable successful, in patients who were assigned to CsA significantly more often a relapse of the nephrotic syndrome occurred than in those who were assigned to the ivCy regimen. Remarkably, race and ethnicity was no independent predictor for remission. Recently, Rahakrishnan et al. analyzed 87 patients with class $\mathrm{V}$ lupus nephritis, who were enrolled in two studies, to assess the efficacy of mycophenolate (MMF) versus ivCy as induction therapy..$^{32 ; 33 ; 42 ; 45}$ Both arms, MMF and ivCy showed improvement of proteinuria, but no significant change of serum creatinine levels at 24 weeks. There was no difference between the MMF and ivCy group regarding percentual change of urinary protein excretion or serum creatinine. The analysis of this reasonable large patient cohort with pure class $V$ LN suggests that both therapies have similar efficacy as induction therapy. However, the end-points were evaluated after 24 weeks. Long-term results are lacking so far which limits the strength of the conclusions.

Encouraging long-term results regarding the use of $A Z A$ as maintenance therapy in pure lupus membranous nephropathy have been reported by Mok et al. ${ }^{46}$ They observed in one-third of patients (13 out of 38 ) renal flares over a long period of 12 years. In summary, this study suggests 
AZA in combination with prednisone as an effective, inexpensive and welltolerated maintenance therapy.

\section{Class VI lupus nephritis}

Class VI LN is characterized by irreversible scarring of renal tissue, without inflammatory activity. Therefore, patients suffering from class VI LN do not benefit from immunosuppressive treatment regimens and, unavoidable, will reach end-stage renal disease (ESRD). In the context of ESRD renal replacement therapies should be discussed such as dialysis or transplantation, as recently reviewed. ${ }^{47}$

\section{Supportive therapy}

Apart from the risk of progressive loss of renal function, various co-morbidities are associated with LN. Moreover, adverse effects due to therapeutic regimens contribute to an increased morbidity in LN patients. Therefore, the reduction of mortality and preservation of renal function remains an important aim. Initially, hydrochloroquine (HCQ) was mainly prescribed for patients with mild lupus manifestations (skin lesions; arthritis). However, a recent meta-analysis revealed that additional treatment with $\mathrm{HCQ}$ decreases disease activity; prevents flares; increases the probability of reaching a renal remission, improves survival, prevents irreversible organ damage and is safe and effective during pregnancy. ${ }^{21}$ Therefore, it seems justified to prescribe HCQ (400 $\mathrm{mg} /$ day) as an adjunct treatment to all patients with $L N$, also in face of its low frequency of side-effects.

Additionally, the reduction of mortality can be approached by several supportive therapeutic measures, which have been recently reviewed by Masood et al. ${ }^{48}$ Briefly, these therapies comprise medications controlling blood pressure, correcting dyslipidemia, preventing thomboembolic events and osteoporosis due to long-term therapy with corticosteroids.

It is well accepted that SLE patients have an increased cardio-vascular risk and therefore blood pressure control is crucial. ${ }^{49 ; 50}$ Blood pressure control 152 
can be ideally achieved by the use of angiotensin-converting enzyme (ACE)inhibitors or angiotensin receptor blockers. ACE- inhibitors have been shown to reduce proteinuria and to delay occurrence of renal involvement of SLEpatients. $^{51}$ Moreover, hyperlipidaemia should be addressed as well. Less evidence is available for the prophylactic long-term anticoagulation of LN patients although nephritis itself is a risk factor for thrombosis. ${ }^{52}$ Anticoagulation with acetylsalicylic acid or acenocoumarol or warfarin should be considered if the renal biopsy demonstrates a thrombotic microangiopathy, which occurs most likely in the context of an antiphospholipid syndrome. However, RCT showing a beneficial effect of long term anticoagulation in LN are lacking so far. Furthermore, the prevention of osteopenia/osteoporosis due to corticosteroids should be addressed by the supplementation of vitamin $D$, calcium and in selected cases of bisphosphonates. In summary, there are several therapeutic approaches which have to be considered, beyond immunosuppression, to treat patients with LN adequately.

\section{Biologicals}

In contrast to the standard immunosuppressive drugs, novel treatments target specifically receptors to inhibit B- and T-cell interaction, to eliminate B-cells or cytokines influencing B- and T-cell activity and function. ${ }^{53}$ Several studies investigated experimental treatments including anti-CD20, anti-CD22, anti-BLyS (B lymphocyte stimulator), CTLA-4-Ig, and abetimus sodium (LJP-394) (Figure 2). 

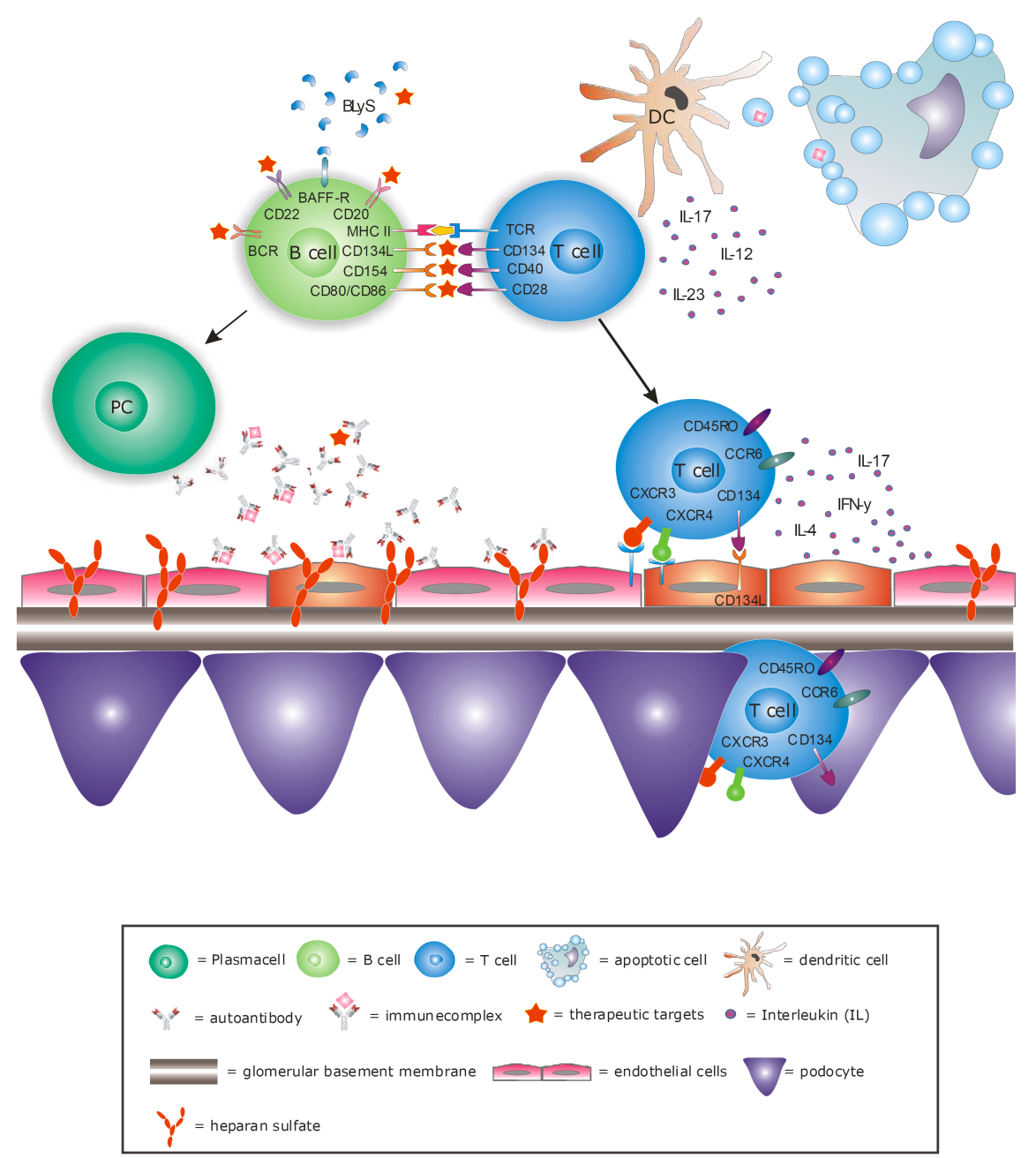

Figure 2: This figure shows the hypothetical role for B- and T-cells for induction of glomerular inflammation in the pathogenesis of lupus nephritis (LN). On the one hand decreased clearance of apoptotic cells can activate dendritic cells (DC) to trigger T-cell response (IL-17 production). On the other hand $\mathrm{B}$ - and T-cells activate each other by interaction and costimulation. Activated B-cells differentiated into plasmacells (PC) are able to produce auto-antibodies which subsequently form antigen/antibody complexes. Both, the antibodies as well as antigen antibody complexes can cause inflammation through deposition at the level of the glomerular basement membrane or bind to basement membrane constituents such as heparan sulfate. Activated effector T-cells might cause tissue injury as well. Chemokine receptors and activation markers enable them to migrate into the kidney. Several therapeutic interventions are illustrated by depletion of B cells, by regulation of Bcell activity, by blocking costimulation and by elimination of circulating auto-antibodies. 
Agents directed against B-cells specifically targeting CD20 (rituximab/ocrelizumab) and CD22 (epratuzumab), have been investigated, since depletion or modulation of B-cells have been shown to be safe and effective in other autoimmune diseases than SLE. ${ }^{54-56}$ The fact that pathogenic autoantibodies in SLE are produced by B-cells raised the idea that depletion of B-cells might be beneficial. Additionally, case reports and single centre experience in LN patients refractory to standard immunosuppression provided encouraging results that indeed B-cell depletion might be effective in the treatment of LN. ${ }^{56}$ However, the results of the first RCT were disappointing. In the LUNAR study patients with LN (class III or IV) were randomized for rituximab (1000 $\mathrm{mg}$ at day 1,15, 168 and 182) or placebo which was given on top of standard of care treatment, being MMF (1500 mg twice daily) and oral prednisone (60 mg initially, with subsequent tapering). ${ }^{57}$ In both arms 72 patients were included. There were no differences in CR or partial remission between both groups $(p=0.55)$. The only significant difference in rituximab treated patients was a larger decrease in anti-dsDNA titers and a more pronounced increase in C3 levels. So, in patients with proliferative LN, addition of rituximab to induction therapy with MMF did not provide better (short-term) results.

The BELONG (NCT00626197) study enrolled SLE patients with biospsy proven class III and IV LN to evaluate whether the addition of the humanized anti-CD20 antibody (ocrelizumab) to standard immunosuppressive care did provide additional benefit. This study was stopped prematurely because of an increased incidence of opportunistic infections. More promising results have been obtained by Dörner et al. who have demonstrated, in an open-label study, comprising 14 lupus patients, improvement of disease activity after epratuzumab (humanized anti-CD22) ${ }^{58}$ In addition to CD20, CD22 is another Bcell restricted receptor, mainly expressed on premature and mature B-cells, but absent on plasma cells. Epratuzumab probably acts via modulation of the B-cell receptor $(B C R)$ and partially through B-cell depletion, but the exact mechanism is unknown so far. In the study by Dörner et al. the administration of 4 
consecutive weekly doses of $360 \mathrm{mg} / \mathrm{m}^{2}$ epratuzumab in SLE patients resulted in clinical improvement as assessed by the BILAG score. Larger trials, confirming these preliminary, data especially with respect to $L N$, are lacking so far.

The impact of B- and T-cell interaction via costimulation is well known. CTLA4Ig is homologue to CD28 and binds to CD80/CD86 with high affinity. Infusion of CTLA4Ig obviously results in downregulation of B cell activity by blocking the CD28-CD80/CD86 pathway. In human trials CTLA4Ig (abatacept) has been successfully evaluated in rheumatoid arthritis. ${ }^{59}$ Results of controlled studies in patients with SLE are currently not available, but are ongoing. A phase-II multicenter trial (NCT00774852, ACCESS study) will evaluate the additional effect of abatacept to the ELNT regimen in LN patients. A benefical effect might be expected, as high levels of co-stimulatory molecules, especially CD80 and CD86 on B cells, were also found in human SLE. ${ }^{11 ; 60}$ In a murine LN model, synergistic effects of abatacept with ivCy have been demonstrated. ${ }^{61}$ Furthermore, expression of CD134L has been shown to be up-regulated in proliferative LN, suggesting a role for the CD134-CD134L pathway in its pathogenesis. CD134 expression was correlated with disease activity and associated with renal involvement in human SLE. ${ }^{12 ; 62}$ A current study in BxBS mice demonstrated that blocking this interaction could be an effective alternative target to attenuate $\mathrm{LN} .^{63}$ This could be a promising future therapeutic target in LN. An antagonist against CD154 (CD40L) has been examined as a possible therapeutic approach in human LN. But short-term administration of anti-CD154 was associated with increased thrombosis, despite initial encouraging data about serology and renal function. ${ }^{64}$

Beside the elimination/modulation of B-cells, also biologicals targeting anti-dsDNA antibodies directly have been investigated. There is substantial evidence that anti-dsDNA antibodies are involved in the pathogenesis of LN. Abetimus sodium (LJP-394) is a synthetic biological agent which is composed of four double stranded oligonucleotides linked to a non immunogenic polyethylene glycol platform. The ratio to use this construct was either as an 156 
antagonist for the B cell receptor for DNA or as a decoy-antigen for anti-dsDNA antibodies. Intravenous administration of abetimus results in a rapid reduction of circulating anti-dsDNA antibody titres. ${ }^{65 ; 66}$ Cardiel et al. investigated whether treatment with abetimus (100 mg/week) for up to 22 months versus placebo could delay renal flares in a cohort of 317 patients. The authors reported a trend in several renal endpoints such as $25 \%$ fewer renal flares in the abetimus arm, but there was no significantly prolonged time to renal flares in these patients. ${ }^{67}$

Regulators of B cell activity such as B lymphocyte stimulator (BLyS) have also been investigated in SLE since elevated serum levels of BLyS have been detected in murine SLE models (MRL/Mp $\left.{ }^{-1 \mathrm{pr} / \mathrm{lpr}}\right)$. In this lupus model serum levels of BLyS seem to be associated with kidney damage whereas treatment with soluble BLyS receptor significantly prolonged survival of lupus mice. ${ }^{68}$ Belimumab is a fully humanized IgG1 monoclonal antibody that binds to soluble human BLyS and inhibits its biologic activity. First results of a phase II trial in SLE patients showed a no beneficial effect of administration belimumab in combination with standard of care medication (SOC) in comparison with SOC alone. $^{69}$ The role of belimumab in LN has still to be elucidated.

Until now, all these agents had no additional beneficial effects in comparison to the "standard of care" immunosuppressive treatment. The lack of data from larger cohorts and long-term follow-up of RCTs hamper the interpretation of the currently available data. Until now there are no data to propagate the standard use of biologicals for induction therapy, but they might be an alternative option in LN patients resistant to other immunosuppressives. This recommendation is based on good results of rituximab therapy reported in (large) case-series of patients with (refractory) LN. ${ }^{70-72}$ The effect of rituximab in the LUNAR study might have gone unnoticed due to the relative mild to moderate lupus disease activity in the patients included. Furthermore, standard of care immunosuppressions is apparently sufficient in the majority of patients. The beneficial effects of rituximab become clear in those patients who have failed on other immunosuppressives. Besides this study design, which_reduced 
the power of the study to detect a favourable response to rituximab in LN, longterm data are necessary. ${ }^{73}$

\section{Expert Commentary \& Five-year view}

In the last decade many studies provided useful information contributing to a better insight into the pathogenesis of SLE and to improvement of therapy. Current treatments appear to be relatively safe and efficacious in a large proportion of SLE patients. However, LN remains a difficult entity among SLE manifestations and therefore the therapeutic regimens are more complex and more toxic. Long term follow-up data are mandatory to define successful responses to treatment and to detect of possible long term adverse effects. Long-term data are available at least for the induction therapy with AZA/MP (Dutch Lupus Nephritis Trial) and low dose ivCy in proliferative LN (EUROLupus trial). Additionally, initial results of alternative induction therapies i.e. with MMF are promising (ALMS-study), but long-term data are essential and can be expected within the next years. On the basis of these studies the following conclusions can be drawn so far:

- for Caucasian patients low dose ivCY (6x500 mg, fortnightly) is an effective induction treatment

- MMF (2x $1.5 \mathrm{~g} /$ day) seems to be an alternative induction treatment, especially for black patients. However, one should realize that longterm data are lacking.

- In patients with severe LN (severe renal impairment/high prevalence of glomerular crescents) high dose IVCY might still be option.

For maintenance treatment limited data are available. Preliminary data of the MAINTAIN study have been published, but final analysis is still pending and can be expected by the end of this year. These data indicate that maintenance treatment with AZA is equal effective as MMF. More difficult remains the interpretation of studies investigating the use of biologicals. This growing group of highly specific immuno-modulators has been studied in addition to standard of care treatment. Recently, RCTs have been performed for anti-CD20 158 
therapies, but other biologicals have been evaluated with other study designs. Therefore, within the next years RCTs including larger cohorts of patients will be initiated. So far data indicate that addition of these biologicals to standard care immunosuppression does not provide additional benefit in induction treatment of proliferative lupus nephritis. With the current status of knowledge it should be reserved for cases refractory to treatment with standard care immunosuppressives. Moreover, in severe refractory LN and life threatening lupus immunoablative chemotherapy followed by autologous hemopoietic stem cell transplantation might be considered. Limited single center experience showed long-term remission in 5/7 patients. On the other hand this extensive treatment was associated with a mortality rate of almost $30 \%{ }^{74} \mathrm{~A}$ less harmful result has been obtained by allogeneic mesenchymal stem cell transplantation (MSCT) in refractory SLE. ${ }^{75}$ In a cohort of 15 SLE patients with persistently active disease a significant reduction in SLEDAI (from 12.2 to 3.2, at 12-month follow-up) and proteinuria (from 2.5 to $0.85 \mathrm{~g} / 24 \mathrm{~h}$ ) was shown. Similar results have been reported by Sun et al. with umbilical cord-derived MSCs in 16 refractory SLE patients. ${ }^{76}$ At present, these regimens remain experimental, but several RCTs evaluating the effect of transplanting autologous or mesenchymal stem cells in SLE patients, are currently carried out.

In conclusion, recent studies have indicated that long-term use of high doses of ivCY are not the first choice treatment option for proliferative lupus nephritis. Low dose ivCY or MMF are now the first options. Long-term data on induction and maintenance treatment will help to define therapeutic decisions more precisely. Moreover, at this moment the various biologicals do not provide extra benefit over the current standard of care. A more precise definition of patients who will benefit from those biologicals, could establish tailor-made therapy. Especially, in cases of refractory LN this new generation of medication will become a valuable alternative option. 


\section{Key Issues}

- Lupus nephritis (LN) is a severe manifestation of systemic lupus erythematosus which should be confirmed by a renal biopsy

- $\mathrm{LN}$ is classified according to the ISN/RPS classification which comprises six classes (I-VI)

- The induction regimen of class III and IV LN includes ivCy (Eurolupus) or MMF in combination with prednisone. The maintenance therapy preferably consists of AZA with prednisone. MMF or CsA might be considered as alternatives whenever AZA is not tolerated.

- $\quad$ Class V LN can be treated with MMF, CsA or ivCy, respectively. The level of initial proteinuria is a good predictor of renal response to treatment.

- The use of biological agents (rituximab) has shown only beneficial effects for selected patients with refractory class III/IV LN

- Supportive care, including use of hydroxychloroquine, is mandatory to further decrease morbidity and mortality in LN patients. 


\section{References}

1. Hahn,B.H. 1998. Antibodies to DNA. N. Engl. J. Med. 338:1359-1368.

2. Doria,A., L.laccarino, A.Ghirardello, S.Zampieri, S.Arienti, P.Sarzi-Puttini, F.Atzeni, A.Piccoli, and S.Todesco. 2006. Long-term prognosis and causes of death in systemic lupus erythematosus. Am. J. Med. 119:700-706.

3. Bono,L., J.S.Cameron, and J.A.Hicks. 1999. The very long-term prognosis and complications of lupus nephritis and its treatment. QJM. 92:211-218.

4. Berden,J.H. 1997. Lupus nephritis. Kidney Int. 52:538-558.

5. Winfield,J.B., I.Faiferman, and D.Koffler. 1977. Avidity of anti-DNA antibodies in serum and IgG glomerular eluates from patients with systemic lupus erythematosus. Association of high avidity antinative DNA antibody with glomerulonephritis. J. Clin. Invest 59:90-96.

6. Pankewycz,O.G., P.Migliorini, and M.P.Madaio. 1987. Polyreactive autoantibodies are nephritogenic in murine lupus nephritis. J. Immunol. 139:3287-3294.

7. van Bruggen,M.C., C.Kramers, M.N.Hylkema, R.J.Smeenk, and J.H.Berden. 1996. Significance of anti-nuclear and anti-extracellular matrix autoantibodies for albuminuria in murine lupus nephritis; a longitudinal study on plasma and glomerular eluates in MRL/I mice. Clin. Exp. Immunol. 105:132-139.

8. Faaber,P., T.P.Rijke, L.B.van de Putte, P.J.Capel, and J.H.Berden. 1986. Crossreactivity of human and murine anti-DNA antibodies with heparan sulfate. The major glycosaminoglycan in glomerular basement membranes. J. Clin. Invest 77:1824-1830.

9. van Bavel,C.C., K.A.Fenton, O.P.Rekvig, J.van der Vlag, and J.H.Berden. 2008. Glomerular targets of nephritogenic autoantibodies in systemic lupus erythematosus. Arthritis Rheum. 58:1892-1899.

10. Nagafuchi,H., Y.Shimoyama, J.Kashiwakura, M.Takeno, T.Sakane, and N.Suzuki. 2003. Preferential expression of B7.2 (CD86), but not B7.1 (CD80), on B cells induced by CD40/CD40L interaction is essential for anti-DNA autoantibody production in patients with systemic lupus erythematosus. Clin. Exp. Rheumatol. 21:71-77.

11. Bijl,M., G.Horst, P.C.Limburg, and C.G.Kallenberg. 2001. Expression of costimulatory molecules on peripheral blood lymphocytes of patients with systemic lupus erythematosus. Ann. Rheum. Dis. 60:523-526.

12. Patschan,S., S.Dolff, A.Kribben, J.Durig, D.Patschan, B.Wilde, C.Specker, T.Philipp, and O.Witzke. 2006. CD134 expression on CD4+ T cells is associated 
with nephritis and disease activity in patients with systemic lupus erythematosus. Clin. Exp. Immunol. 145:235-242.

13. Enghard,P., J.Y.Humrich, B.Rudolph, S.Rosenberger, R.Biesen, A.Kuhn, R.Manz, F.Hiepe, A.Radbruch, G.R.Burmester, and G.Riemekasten. 2009. CXCR3+CD4+ $\mathrm{T}$ cells are enriched in inflamed kidneys and urine and provide a new biomarker for acute nephritis flares in systemic lupus erythematosus patients. Arthritis Rheum. 60:199-206.

14. Dolff,S., W.H.Abdulahad, M.C.van Dijk, P.C.Limburg, C.G.Kallenberg, and M.Bijl. 2010. Urinary $T$ cells in active lupus nephritis show an effector memory phenotype. Ann. Rheum. Dis. 69:2034-2041.

15. Houssiau,F.A., D.D'Cruz, J.Vianna, and G.R.Hughes. 1991. Lupus nephritis: the significance of serological tests at the time of biopsy. Clin. Exp. Rheumatol. 9:345349.

16. Hill,G.S., N.Hinglais, F.Tron, and J.F.Bach. 1978. Systemic lupus erythematosus. Morphologic correlations with immunologic and clinical data at the time of biopsy. Am. J. Med. 64:61-79.

17. Esdaile,J.M., C.Levinton, W.Federgreen, J.P.Hayslett, and M.Kashgarian. 1989. The clinical and renal biopsy predictors of long-term outcome in lupus nephritis: a study of 87 patients and review of the literature. Q. J. Med. 72:779-833.

18. Weening,J.J., V.D.D'Agati, M.M.Schwartz, S.V.Seshan, C.E.Alpers, G.B.Appel, J.E.Balow, J.A.Bruijn, T.Cook, F.Ferrario, A.B.Fogo, E.M.Ginzler, L.Hebert, G.Hill, P.Hill, J.C.Jennette, N.C.Kong, P.Lesavre, M.Lockshin, L.M.Looi, H.Makino, L.A.Moura, and M.Nagata. 2004. The classification of glomerulonephritis in systemic lupus erythematosus revisited. Kidney Int. 65:521-530.

19. Austin,H.A., III, L.R.Muenz, K.M.Joyce, T.T.Antonovych, and J.E.Balow. 1984. Diffuse proliferative lupus nephritis: identification of specific pathologic features affecting renal outcome. Kidney Int. 25:689-695.

20. Austin,H.A., III, D.T.Boumpas, E.M.Vaughan, and J.E.Balow. 1994. Predicting renal outcomes in severe lupus nephritis: contributions of clinical and histologic data. Kidney Int. 45:544-550.

21. Ruiz-Irastorza,G., M.Ramos-Casals, P.Brito-Zeron, and M.A.Khamashta. 2010. Clinical efficacy and side effects of antimalarials in systemic lupus erythematosus: a systematic review. Ann. Rheum. Dis. 69:20-28.

22. Grootscholten,C. and J.H.Berden. 2006. Discontinuation of immunosuppression in proliferative lupus nephritis: is it possible? Nephrol. Dial. Transplant. 21:14651469. 
23. Steinberg,A.D. and S.C.Steinberg. 1991. Long-term preservation of renal function in patients with lupus nephritis receiving treatment that includes cyclophosphamide versus those treated with prednisone only. Arthritis Rheum. 34:945-950.

24. Gourley,M.F., H.A.Austin, III, D.Scott, C.H.Yarboro, E.M.Vaughan, J.Muir, D.T.Boumpas, J.H.Klippel, J.E.Balow, and A.D.Steinberg. 1996. Methylprednisolone and cyclophosphamide, alone or in combination, in patients with lupus nephritis. A randomized, controlled trial. Ann. Intern. Med. 125:549557.

25. Boumpas,D.T., H.A.Austin, III, E.M.Vaughn, J.H.Klippel, A.D.Steinberg, C.H.Yarboro, and J.E.Balow. 1992. Controlled trial of pulse methylprednisolone versus two regimens of pulse cyclophosphamide in severe lupus nephritis. Lancet 340:741-745.

26. Houssiau,F.A., C.Vasconcelos, D.D'Cruz, G.D.Sebastiani, R.Garrido Ed Ede, M.G.Danieli, D.Abramovicz, D.Blockmans, A.Mathieu, H.Direskeneli, M.Galeazzi, A.Gul, Y.Levy, P.Petera, R.Popovic, R.Petrovic, R.A.Sinico, R.Cattaneo, J.Font, G.Depresseux, J.P.Cosyns, and R.Cervera. 2002. Immunosuppressive therapy in lupus nephritis: the Euro-Lupus Nephritis Trial, a randomized trial of low-dose versus high-dose intravenous cyclophosphamide. Arthritis Rheum. 46:2121-2131.

27. Houssiau,F.A., C.Vasconcelos, D.D'Cruz, G.D.Sebastiani, E.Ramon Garrido, M.G.Danieli, D.Abramovicz, D.Blockmans, A.Cauli, H.Direskeneli, M.Galeazzi, A.Gul, Y.Levy, P.Petera, R.Popovic, R.Petrovic, R.A.Sinico, R.Cattaneo, J.Font, G.Depresseux, J.P.Cosyns, and R.Cervera. 2010. The 10-year follow-up data of the Euro-Lupus Nephritis Trial comparing low-dose and high-dose intravenous cyclophosphamide. Ann. Rheum. Dis. 69:61-64.

28. Grootscholten,C., G.Ligtenberg, E.C.Hagen, van den Wall Bake AW, J.W.GlasVos, M.Bijl, K.J.Assmann, J.A.Bruijn, J.J.Weening, H.C.van Houwelingen, R.H.Derksen, and J.H.Berden. 2006. Azathioprine/methylprednisolone versus cyclophosphamide in proliferative lupus nephritis. A randomized controlled trial. Kidney Int. 70:732-742.

29. Grootscholten,C., I.M.Bajema, S.Florquin, E.J.Steenbergen, C.J.Peutz-Kootstra, R.Goldschmeding, M.Bijl, E.C.Hagen, H.C.van Houwelingen, R.H.Derksen, and J.H.Berden. 2007. Treatment with cyclophosphamide delays the progression of chronic lesions more effectively than does treatment with azathioprine plus methylprednisolone in patients with proliferative lupus nephritis. Arthritis Rheum. 56:924-937. 
30. Chan,T.M., F.K.Li, C.S.Tang, R.W.Wong, G.X.Fang, Y.L.Ji, C.S.Lau, A.K.Wong, M.K.Tong, K.W.Chan, and K.N.Lai. 2000. Efficacy of mycophenolate mofetil in patients with diffuse proliferative lupus nephritis. Hong Kong-Guangzhou Nephrology Study Group. N. Engl. J. Med. 343:1156-1162.

31. Chan,T.M., K.C.Tse, C.S.Tang, M.Y.Mok, and F.K.Li. 2005. Long-term study of mycophenolate mofetil as continuous induction and maintenance treatment for diffuse proliferative lupus nephritis. J. Am. Soc. Nephrol. 16:1076-1084.

32. Ginzler,E.M., M.A.Dooley, C.Aranow, M.Y.Kim, J.Buyon, J.T.Merrill, M.Petri, G.S.Gilkeson, D.J.Wallace, M.H.Weisman, and G.B.Appel. 2005. Mycophenolate mofetil or intravenous cyclophosphamide for lupus nephritis. N. Engl. J. Med. 353:2219-2228.

33. Appel,G.B., G.Contreras, M.A.Dooley, E.M.Ginzler, D.Isenberg, D.Jayne, L.S.Li, E.Mysler, J.Sanchez-Guerrero, N.Solomons, and D.Wofsy. 2009. Mycophenolate mofetil versus cyclophosphamide for induction treatment of lupus nephritis. J. Am. Soc. Nephrol. 20:1103-1112.

34. Bao,H., Z.H.Liu, H.L.Xie, W.X.Hu, H.T.Zhang, and L.S.Li. 2008. Successful treatment of class $\mathrm{V}+\mathrm{IV}$ lupus nephritis with multitarget therapy. J. Am. Soc. Nephrol. 19:2001-2010.

35. Isenberg,D., G.B.Appel, G.Contreras, M.A.Dooley, E.M.Ginzler, D.Jayne, J.Sanchez-Guerrero, D.Wofsy, X.Yu, and N.Solomons. 2010. Influence of race/ethnicity on response to lupus nephritis treatment: the ALMS study. Rheumatology. (Oxford) 49:128-140.

36. Moroni,G., A.Doria, M.Mosca, O.D.Alberighi, G.Ferraccioli, S.Todesco, C.Manno, P.Altieri, R.Ferrara, S.Greco, and C.Ponticelli. 2006. A randomized pilot trial comparing cyclosporine and azathioprine for maintenance therapy in diffuse lupus nephritis over four years. Clin. J. Am. Soc. Nephrol. 1:925-932.

37. Contreras,G., V.Pardo, B.Leclercq, O.Lenz, E.Tozman, P.O'Nan, and D.Roth. 2004. Sequential therapies for proliferative lupus nephritis. N. Engl. J. Med. 350:971-980.

38. Houssiau FA, D'Cruz DP, and Sangle SR. 2009. Azathioprine Versus Mycophenolate Mofetil for Maintenance Immunosuppression of Proliferative Lupus Nephritis: Results of a Randomized Trial (MAINTAIN); Presented at $73^{\text {th }}$ Annual Scientific Meeting of the American College of Rheumatology, October 19, 2009; Philadelphia, PA. Arthritis Rheum.

39. Zhu,B., N.Chen, Y.Lin, H.Ren, W.Zhang, W.Wang, X.Pan, and H.Yu. 2007. Mycophenolate mofetil in induction and maintenance therapy of severe lupus 
nephritis: a meta-analysis of randomized controlled trials. Nephrol. Dial. Transplant. 22:1933-1942.

40. Bhinder,S., A.Singh, and V.Majithia. 2010. Membranous (class V) renal disease in systemic lupus erythematosus may be more common than previously reported: results of a 6-year retrospective analysis. Am. J. Med. Sci. 339:230-232.

41. Moroni,G., M.Maccario, G.Banfi, S.Quaglini, and C.Ponticelli. 1998. Treatment of membranous lupus nephritis. Am. J. Kidney Dis. 31:681-686.

42. Radhakrishnan,J., C.L.Kunis, V.D'Agati, and G.B.Appel. 1994. Cyclosporine treatment of lupus membranous nephropathy. Clin. Nephrol. 42:147-154.

43. Mercadal,L., S.T.Montcel, D.Nochy, G.Queffeulou, J.C.Piette, C.Isnard-Bagnis, and F.Martinez. 2002. Factors affecting outcome and prognosis in membranous lupus nephropathy. Nephrol. Dial. Transplant. 17:1771-1778.

44. Austin,H.A., III, G.G.Illei, M.J.Braun, and J.E.Balow. 2009. Randomized, controlled trial of prednisone, cyclophosphamide, and cyclosporine in lupus membranous nephropathy. J. Am. Soc. Nephrol. 20:901-911.

45. Radhakrishnan,J., D.A.Moutzouris, E.M.Ginzler, N.Solomons, I.I.Siempos, and G.B.Appel. 2010. Mycophenolate mofetil and intravenous cyclophosphamide are similar as induction therapy for class $\vee$ lupus nephritis. Kidney Int. 77:152-160.

46. Mok,C.C., K.Y.Ying, C.W.Yim, W.L.Ng, and W.S.Wong. 2009. Very long-term outcome of pure lupus membranous nephropathy treated with glucocorticoid and azathioprine. Lupus 18:1091-1095.

47. Rietveld,A. and J.H.Berden. 2008. Renal replacement therapy in lupus nephritis. Nephrol. Dial. Transplant. 23:3056-3060.

48. Masood,S., D.Jayne, and Y.Karim. 2009. Beyond immunosuppression challenges in the clinical management of lupus nephritis. Lupus 18:106-115.

49. de Leeuw,K., B.Freire, A.J.Smit, H.Bootsma, C.G.Kallenberg, and M.Bijl. 2006. Traditional and non-traditional risk factors contribute to the development of accelerated atherosclerosis in patients with systemic lupus erythematosus. Lupus 15:675-682.

50. de Leeuw,K., A.J.Smit, E.de Groot, A.M.van Roon, C.G.Kallenberg, and M.Bijl. 2009. Longitudinal study on premature atherosclerosis in patients with systemic lupus erythematosus. Atherosclerosis 206:546-550.

51. Duran-Barragan,S., G.McGwin, Jr., L.M.Vila, J.D.Reveille, and G.S.Alarcon. 2008. Angiotensin-converting enzyme inhibitors delay the occurrence of renal involvement and are associated with a decreased risk of disease activity in 
patients with systemic lupus erythematosus--results from LUMINA (LIX): a multiethnic US cohort. Rheumatology. (Oxford) 47:1093-1096.

52. Kaiser,R., C.M.Cleveland, and L.A.Criswell. 2009. Risk and protective factors for thrombosis in systemic lupus erythematosus: results from a large, multi-ethnic cohort. Ann. Rheum. Dis. 68:238-241.

53. Bhat,P. and J.Radhakrishnan. 2008. B lymphocytes and lupus nephritis: new insights into pathogenesis and targeted therapies. Kidney Int. 73:261-268.

54. Emery,P., A.Deodhar, W.F.Rigby, J.D.Isaacs, B.Combe, A.J.Racewicz, K.Latinis, C.Abud-Mendoza, L.J.Szczepanski, R.A.Roschmann, A.Chen, G.K.Armstrong, W.Douglass, and H.Tyrrell. 2010. Efficacy and safety of different doses and retreatment of rituximab: a randomised, placebo-controlled trial in patients who are biological naive with active rheumatoid arthritis and an inadequate response to methotrexate (Study Evaluating Rituximab's Efficacy in MTX iNadequate rEsponders (SERENE)). Ann. Rheum. Dis.

55. Meijer,J.M., J.Pijpe, A.Vissink, C.G.Kallenberg, and H.Bootsma. 2009. Treatment of primary Sjogren syndrome with rituximab: extended follow-up, safety and efficacy of retreatment. Ann. Rheum. Dis. 68:284-285.

56. Ramos-Casals,M., C.Diaz-Lagares, M.J.Soto-Cardenas, P.Brito-Zeron, M.J.Cuadrado, G.Sanna, L.Bertolaccini, and M.A.Khamashta. 2010. Rituximab Therapy in Lupus Nephritis: Current Clinical Evidence. Clin. Rev. Allergy Immunol.

57. Furie R, Looney RJ, and Rovin B. 2009. Efficacy and Safety of Rituximab in Subjects with Active Proliferative Lupus Nephritis (LN): Results From the Randomized, Double-Blind Phase III LUNAR Study. Presented at $73^{\text {th }}$ Annual Scientific Meeting of the American College of Rheumatology, October 19, 2009; Philadelphia, PA. Arthritis Rheum.

58. Dorner,T., J.Kaufmann, W.A.Wegener, N.Teoh, D.M.Goldenberg, and G.R.Burmester. 2006. Initial clinical trial of epratuzumab (humanized anti-CD22 antibody) for immunotherapy of systemic lupus erythematosus. Arthritis Res. Ther. 8:R74.

59. Kremer,J.M., H.K.Genant, L.W.Moreland, A.S.Russell, P.Emery, C.AbudMendoza, J.Szechinski, T.Li, J.Teng, J.C.Becker, and R.Westhovens. 2008. Results of a two-year followup study of patients with rheumatoid arthritis who received a combination of abatacept and methotrexate. Arthritis Rheum. 58:953963.

60. Dolff,S., B.Wilde, S.Patschan, J.Durig, C.Specker, T.Philipp, A.Kribben, and O.Witzke. 2007. Peripheral circulating activated b-cell populations are associated 
with nephritis and disease activity in patients with systemic lupus erythematosus. Scand. J. Immunol. 66:584-590.

61. Daikh,D.I. and D.Wofsy. 2001. Cutting edge: reversal of murine lupus nephritis with CTLA4Ig and cyclophosphamide. J. Immunol. 166:2913-2916.

62. Aten,J., A.Roos, N.Claessen, E.J.Schilder-Tol, I.J.Ten Berge, and J.J.Weening. 2000. Strong and selective glomerular localization of CD134 ligand and TNF receptor-1 in proliferative lupus nephritis. J. Am. Soc. Nephrol. 11:1426-1438.

63. Zhou,Y.B., R.G.Ye, Y.J.Li, and C.M.Xie. 2009. Targeting the CD134-CD134L interaction using anti-CD134 and/or rhCD134 fusion protein as a possible strategy to prevent lupus nephritis. Rheumatol. Int. 29:417-425.

64. Boumpas,D.T., R.Furie, S.Manzi, G.G.Illei, D.J.Wallace, J.E.Balow, and A.Vaishnaw. 2003. A short course of BG9588 (anti-CD40 ligand antibody) improves serologic activity and decreases hematuria in patients with proliferative lupus glomerulonephritis. Arthritis Rheum. 48:719-727.

65. Weisman,M.H., H.G.Bluestein, C.M.Berner, and H.A.de Haan. 1997. Reduction in circulating dsDNA antibody titer after administration of LJP 394. J. Rheumatol. 24:314-318.

66. Furie,R.A., J.M.Cash, M.E.Cronin, R.S.Katz, M.H.Weisman, C.Aranow, M.R.Liebling, N.P.Hudson, C.M.Berner, S.Coutts, and H.A.de Haan. 2001. Treatment of systemic lupus erythematosus with LJP 394. J. Rheumatol. 28:257265.

67. Cardiel,M.H., J.A.Tumlin, R.A.Furie, D.J.Wallace, T.Joh, and M.D.Linnik. 2008. Abetimus sodium for renal flare in systemic lupus erythematosus: results of a randomized, controlled phase III trial. Arthritis Rheum. 58:2470-2480.

68. Gross,J.A., J.Johnston, S.Mudri, R.Enselman, S.R.Dillon, K.Madden, W.Xu, J.Parrish-Novak, D.Foster, C.Lofton-Day, M.Moore, A.Littau, A.Grossman, H.Haugen, K.Foley, H.Blumberg, K.Harrison, W.Kindsvogel, and C.H.Clegg. 2000. TACl and BCMA are receptors for a TNF homologue implicated in B-cell autoimmune disease. Nature 404:995-999.

69. Wallace,D.J., W.Stohl, R.A.Furie, J.R.Lisse, J.D.McKay, J.T.Merrill, M.A.Petri, E.M.Ginzler, W.W.Chatham, W.J.McCune, V.Fernandez, M.R.Chevrier, Z.J.Zhong, and W.W.Freimuth. 2009. A phase II, randomized, double-blind, placebo-controlled, dose-ranging study of belimumab in patients with active systemic lupus erythematosus. Arthritis Rheum. 61:1168-1178.

70. Gunnarsson,I., B.Sundelin, T.Jonsdottir, S.H.Jacobson, E.W.Henriksson, and R.F.van Vollenhoven. 2007. Histopathologic and clinical outcome of rituximab 
treatment in patients with cyclophosphamide-resistant proliferative lupus nephritis.

Arthritis Rheum. 56:1263-1272.

71. Terrier,B., Z.Amoura, P.Ravaud, E.Hachulla, R.Jouenne, B.Combe, C.Bonnet, P.Cacoub, A.Cantagrel, M.de Bandt, O.Fain, B.Fautrel, P.Gaudin, B.Godeau, J.R.Harle, A.Hot, J.E.Kahn, O.Lambotte, C.Larroche, J.Leone, O.Meyer, B.PallotPrades, E.Pertuiset, P.Quartier, T.Schaerverbeke, J.Sibilia, A.Somogyi, M.Soubrier, E.Vignon, B.Bader-Meunier, X.Mariette, and J.E.Gottenberg. 2010. Safety and efficacy of rituximab in systemic lupus erythematosus: results from 136 patients from the French Autolmmunity and Rituximab registry. Arthritis Rheum. 62:2458-2466.

72. Ramos-Casals,M., M.J.Soto, M.J.Cuadrado, and M.A.Khamashta. 2009. Rituximab in systemic lupus erythematosus: A systematic review of off-label use in 188 cases. Lupus 18:767-776.

73. Ramos-Casals,M., C.Diaz-Lagares, and M.A.Khamashta. 2009. Rituximab and lupus: good in real life, bad in controlled trials. Comment on the article by Lu et al. Arthritis Rheum. 61:1281-1282.

74. Alexander,T., A.Thiel, O.Rosen, G.Massenkeil, A.Sattler, S.Kohler, H.Mei, H.Radtke, E.Gromnica-Ihle, G.R.Burmester, R.Arnold, A.Radbruch, and F.Hiepe. 2009. Depletion of autoreactive immunologic memory followed by autologous hematopoietic stem cell transplantation in patients with refractory SLE induces long-term remission through de novo generation of a juvenile and tolerant immune system. Blood 113:214-223.

75. Liang,J., H.Zhang, B.Hua, H.Wang, L.Lu, S.Shi, Y.Hou, X.Zeng, G.S.Gilkeson, and L.Sun. 2010. Allogenic mesenchymal stem cells transplantation in refractory systemic lupus erythematosus: a pilot clinical study. Ann. Rheum. Dis. 69:14231429.

76. Sun,L., D.Wang, J.Liang, H.Zhang, X.Feng, H.Wang, B.Hua, B.Liu, S.Ye, X.Hu, W.Xu, X.Zeng, Y.Hou, G.S.Gilkeson, R.M.Silver, L.Lu, and S.Shi. 2010. Umbilical cord mesenchymal stem cell transplantation in severe and refractory systemic lupus erythematosus. Arthritis Rheum. 62:2467-2475. 